Journal of Computer Science 7 (3): 416-420, 2011

ISSN 1549-3636

(C) 2011 Science Publications

\title{
Genetic Algorithm Based Proportional Integral Controller Design for Induction Motor
}

\author{
${ }^{1}$ Mohanasundaram Kuppusamy and ${ }^{2}$ Rajasekar Natarajan \\ ${ }^{1}$ Department of Electrical and Electronics Engineering, \\ Sree Sastha Institute of Engineering and Technology, Chembarambakkam, \\ Chennai, 600123, India \\ ${ }^{2}$ School of Electrical Sciences, VIT University, Vellore, Tamilnadu, India
}

\begin{abstract}
Problem statement: This study has expounded the application of evolutionary computation method namely Genetic Algorithm (GA) for estimation of feedback controller parameters for induction motor. GA offers certain advantages such as simple computational steps, derivative free optimization, reduced number of iterations and assured near global optima. The development of the method is well documented and computed and measured results are presented. Approach: The design of PI controller parameter for three phase induction motor drives was done using Genetic Algorithm. The objective function of motor current reduction, using PI controller, at starting is formulated as an optimization problem and solved with Genetic Algorithm. Results: The results showed the selected values of PI controller parameter using genetic algorithm approach, with objective of induction motor starting current reduction. Conclusions/Recommendation: The results proved the robustness and easy implementation of genetic algorithm selection of PI parameters for induction motor starting.
\end{abstract}

Key words: Genetic algorithm, induction motor, evolutionary computation, controller parameters

\section{INTRODUCTION}

The squirrel cage induction motor is basically a simple, less costly and reliable drive and can provide excellent characteristics at a constant shaft speed. Probably the cheapest and most reliable scheme of speed control of induction motors is stator voltage control using back-to-back connected SCRs. This scheme is widely used for certain types of loads such as fan and pump drives. It was shown that speed ranges of 5-1 can be easily obtained using this method (Paice, 1968; Lipo, 1971). However, little attention is being paid to the design of controller for closed loop variable speed operation using the above scheme. The drawback of is that an analytical relationship could not be established between induction motor torque and applied voltage with thyristor excitation and only an empirical approach was used (Shepherd and Stanway, 1967). Further, motor parameter variation with different operating points is not considered.

Genetic Algorithm is a stochastic search techniques based on the mechanism of natural selection and natural genetics. GA (Kangrang and Chaleeraktrakoon, 2007) perform search for a multidimensional space containing a hyper surface known as the fitness surface.
There are many advantages that make GA attractive. GA does not require the use of derivatives. They offer a parallel searching of the solution space rather than the point-by-point searching in a small region (Wieczorek et al., 1998). Since the location of the optimal solution is unknown before the search, it is very likely that the point-by-point search needs to search through the entire solution space in order to find the optimal solution, not mention the traps of the local optima. Hence, GA can find near-optimal solution for a complex problem very quickly.

Problem formulation: Induction motor starting at rated current (Say, 1958) is formulated as an optimization problem and is given below:

Minimize:

$\mathrm{F}(\phi)=|\operatorname{Ir}-\mathrm{I}|$

Subjected to:

$\phi_{\min } \leq \phi \leq \phi_{\max }$

Where:

$\mathrm{Ir}=\quad$ rated motor current

Corresponding Author: Mohanasundaram Kuppusamy, Department of Electrical and Electronics Engineering, Sree Sastha Institute of Engineering and Technology, Chembarambakkam, Chennai, 600123, India Tel: +91 9600047399 
$\mathrm{I}=$ motor current

$\Phi_{\mathrm{s}}=\quad\left\{\mathrm{K}_{\mathrm{p}}, \mathrm{K}_{\mathrm{i}}\right\}$

$\phi_{\min }$ and $\phi_{\max }=$ refer to minimum and maximums of $\phi$

For the evaluation of objective function $\mathrm{F}(\phi)$, the MATLAB model of induction motor is developed to work in the closed loop starting mode (Sundaram et al., 2009) and this is shown in the Fig. 1. Here, PI controller block (Hassanzadeh et al., 2008) refers to controller constants to be identified.

Implementation of GA for optimization: The primary concept of GA was first proposed by Dr. Holland in 1975. GA constitutes biologically inspired multiparameter search/optimization algorithms (Subramanian et al., 2010) that have proven to be effective in solving a variety of complex problems where other algorithms have either failed or faced difficulties.

The following steps describe how the GA is designed and applied to the present problem. Various components of GA (Wurtz et al., 1997) such as chromosomes, fitness function, reproduction, crossover and mutation as applied to the present work are illustrated.

Step1: Create a population of initial solution of parameters $\left(\mathbf{k}_{\mathbf{p}}, \mathbf{k}_{\mathbf{i}}\right)$ : This step primarily requires the population size. Each variable in the problem is called as a gene and in the present problem, there are two (i.e., $\mathrm{k}_{\mathrm{p}}, \mathrm{k}_{\mathrm{i}}$ ) genes. A Chromosome consists of the genes and thus each chromosome represents a solution to the problem. This is illustrated in Fig. 2.

The population consists of a set of chromosomes. It is well articulated in literature that a population size of 5-30 is an ideal one and hence population size is selected as 8 in this study.

Step 2: Evaluation of objective function: In the present problem, the starting current of induction motor is to be maintained at rated value while starting. For each chromosome, the MATLAB model given in Fig. 1. is simulated and $F((\phi))$ is computed.

Step 3: Evaluation of fitness function: The degree of "goodness" of a solution is qualified by assigning a value to it. This is done by defining a proper fitness function to the problem. Since GA can be used only for maximization problems, the following fitness function is used:

Fitness function $=1 /(1+\mathrm{F}(\phi))$

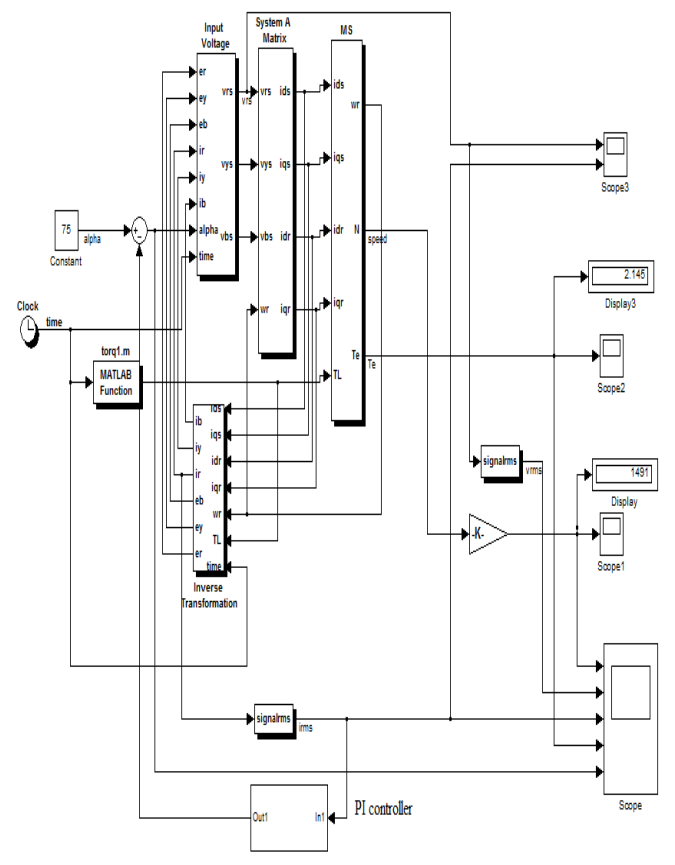

Fig. 1: Matlab/Simulink model of A.C. voltage controller fed induction motor with PI controller

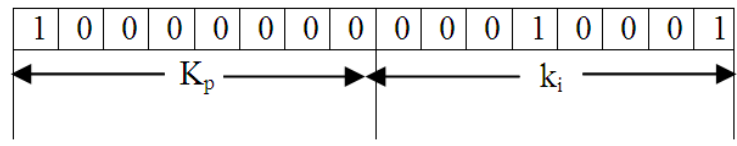

Fig. 2: Chromosome structure

Step 4: Generation of offspring: Offspring is a new chromosome obtained through the steps of selection, crossover and mutation. After fitness of each chromosome is computed, parent solutions are selected for reproduction. It emulates the survival of the fittest mechanism in nature. The Roulette wheel selection is the most common and easy-to-implement selection mechanism. A virtual wheel is implemented for this selection process. Each chromosome is assigned a sector in this virtual wheel and the area of the sector is proportional to their fitness value. Thus the chromosome with largest fitness value will occupy largest area, while the chromosome with a lower value takes the slot of a smaller sector. Let there be five chromosomes labeled as A, B, C, D and E and their fitness value increases in the order of D, B, A, E and C. Then Fig. 3 shows a typical allocation of five sectors of chromosomes in the Roulette wheel.

In Roulette wheel selection, an angle is generated randomly and the chromosome corresponding to this angle is selected. Figure $3 \mathrm{~b}$ shows a randomly 


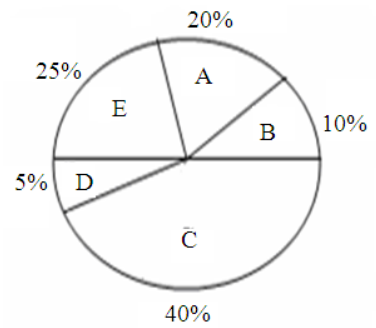

(a)

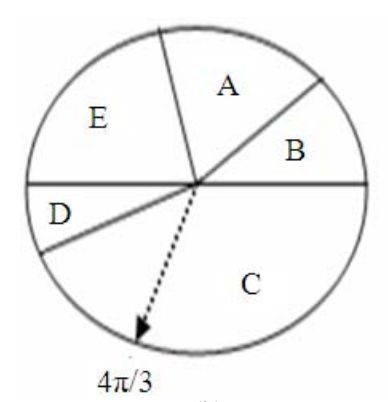

(b)

Fig. 3: (a) Typical allocation of sectors for Chromosomes; (b) Roulette wheel selection

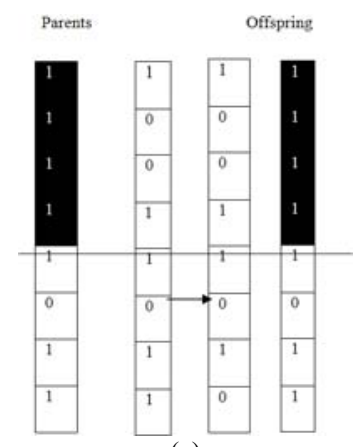

(a)

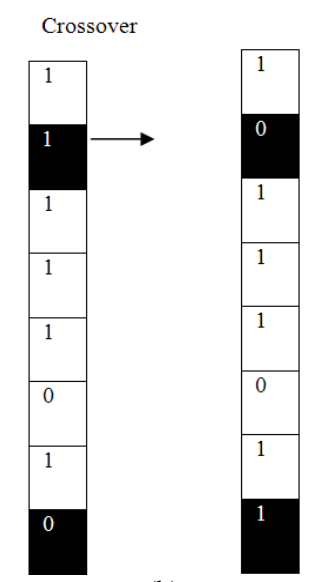

(b)

Fig. 4: (a) Crossover and (b) mutation generated angle of $4 \pi / 3 \mathrm{rad}$. In this case, chromosome $\mathrm{C}$ is selected. The chromosomes thus selected are called parent population and are subjected to undergo crossover and mutation to produce offspring for the next generation. Roulette wheel selection is the conventional method adopted in GA for selection. Following the selection of parent population, crossover and mutation are performed to generate offspring population. In crossover, randomly selected subsections of two individual chromosomes are swapped to produce the offspring. In this study, single point crossover is used for children generation.

Mutation is another genetic operation by which a bit within a chromosome may toggle to the opposite binary. Figure 4 illustrates crossover and mutation. The crossover and mutation are performed based on the probability of crossover and mutation.

Step5: Replace the current population with the new population.

Step6: Terminate the program if termination criterion is reached; else go to step 2

\section{RESULTS}

For the implementation of GA to the present problem, dedicated software in MATLAB is developed. The parameters of GA such as crossover probability, mutation probability, population size and number of generations are usually selected by means of trial and error process to achieve the best solution set. The parameters used in the implementation of GA are listed below:

Population size: $\quad 8$

Coding: Binary

Number of generations: 80

Selection scheme: $\quad$ Roulette wheel

Selection

Crossover operator: Single point

Crossover

Crossover probability: $\quad 0.7$

Mutation probability: $\quad 0.01$

Termination criterion: $\quad 80$ iterations.

Dedicated software is developed in MATLAB for this optimization problem and the results are analyzed. The convergence characteristics with GA are plotted in Fig. 5 and 6 . It can be seen that the objective function value converges to a reasonably low value of 0.03 against its ideal value of zero at 45th iteration. 


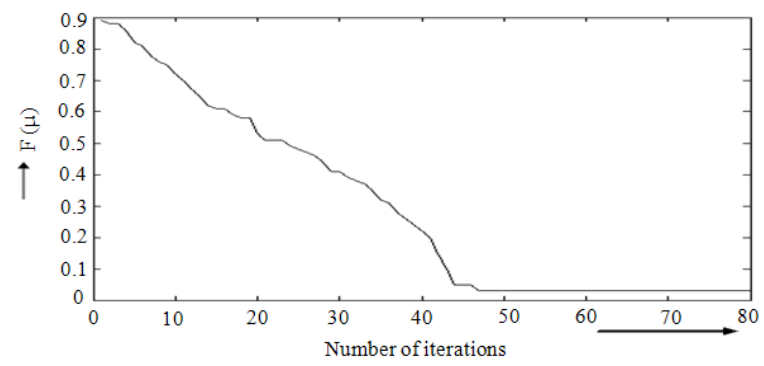

Fig. 5: Variation of objective function

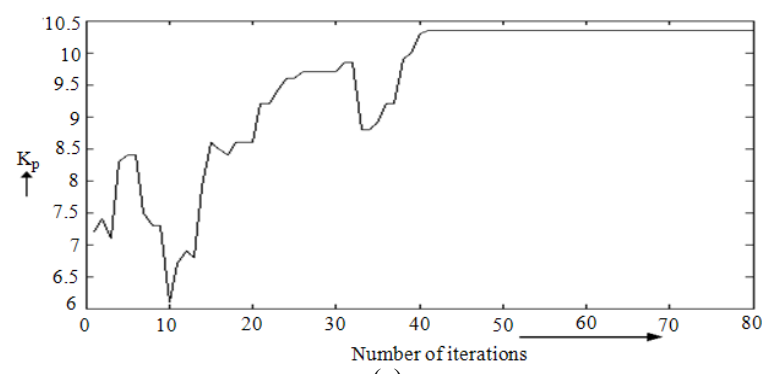

(a)

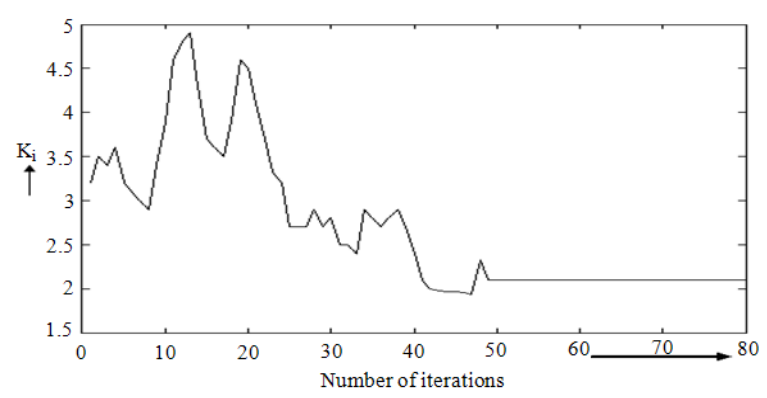

(b)

Fig. 6: Variation of parameters (a) $\mathrm{K}_{\mathrm{p}}$; (b) $\mathrm{K}_{\mathrm{i}}$

The convergence is observed to be at a stable and faster rate. The variation of controller parameters is delineated in Fig. 6.

\section{DISCUSSION}

It is interesting to study the convergence behavior of GA as given in Fig. 6. It is seen that, the number of iterations required for all chromosomes to reach this near-global optima is reasonably low. It is also seen that the parameters of controller are suitably modified at iterative step with the sole aim of minimizing the objective function value. At the end of 50 generations, the constants of controller are obtained as:

$\mathrm{K}_{\mathrm{p}}=10.3$ and $\mathrm{K}_{\mathrm{i}}=2.2$
With these controller constants, induction motor starting is simulated using MATLAB model shown in Fig. 1. It is seen that the motor current is almost kept constant at its rated value during starting. This demonstrates the effectiveness of the proposed approach.

\section{CONCLUSION}

The main objective of this study is tuning of the PI controller parameters by Genetic algorithm. Simulation results with PI controller are included. The parameters of PI controller are tuned using the proposed optimization algorithm.

\section{REFERENCES}

Hassanzadeh, I., S. Mobayen and A. Harifi, 2008. Input-output feedback linearization cascade controller using genetic algorithm for rotary inverted pendulum system. Am. J. Applied Sci., 5: 1322-1328. DOI: 10.3844/ajassp.2008.1322.1328

Kangrang, A. and C. Chaleeraktrakoon, 2007. Genetic algorithms connected simulation with smoothing function for searching rule curves. Am. J. Applied Sci., 4: 73-79. DOI: 10.3844/ajassp.2007.73.79

Lipo, T.A., 1971. The analysis of induction motors with voltage control by symmetrically triggered thyristors. IEEE Trans. Power Apparatus Syst., 90: 515-525. DOI: 10.1109/TPAS.1971.293053

Paice, D.A., 1968. Induction motor speed control by stator voltage control. IEEE Trans. Power Apparatus Syst., 87: 585-590. DOI: 10.1109/TPAS.1968.292056

Say, M.G., 1958. The Performance and Design of Alternating Current Machines: Transformers Three-Phase Induction Motors and Synchronous Machines. 3rd Edn., English Language Book Society, London, pp: 664.

Shepherd, W. and J. Stanway, 1967. An experimental closed-loop variable speed drive incorporating a thyristor driven induction motor. IEEE Trans. Ind. General Appl., 3: 559-565. DOI: 10.1109/TIGA.1967.4180833

Subramanian, R., S.N. Sivanandam and C. Vimalarani, 2010. An optimization of design for s4-duty induction motor using constraints normalization based violation technique. J. Comput. Sci., 6: 107111. DOI: $10.3844 /$ jcssp.2010.107.111

Sundaram, K.M., N. Rajasekar, J.B. Edward and G. Saravanailango, 2009. A Fuzzy logic approach for speed controller design of A.C. voltage controller fed induction motor drive. Proceedings of the 5th International Conference on MEMS, NANO, and Smart Systems, Dec. 28-30, Dubai, United Arab Emirates, pp: 133-136. DOI: 10.1109/ICMENS.2009.26 
Wieczorek, J.P., G. Ozdeir and M. Zbigniew, 1998. An evolutionary algorithm for the optimal design of induction motors. IEEE Trans. Mag., 34: 3882-3887. DOI: $10.1109 / 20.728298$
Wurtz, F., M. Richomme, J. Bigeon and J.C. Sabonnadiere, 1997. A few results for using genetic algorithms in the design of electrical machines. IEEE Trans. Mag., 33: 1892-1895. DOI: 10.1109/20.582656 